null • Business of Knowing, summer 2021

\title{
Open Grant Proposals
}

David Lang

Published on: Aug 31, 2021

License: Creative Commons Attribution 4.0 International License (CC-BY 4.0). 
Science is designed to move slowly. Debate, rigor, and peer review add layers of organized skepticism to new ideas and claims. The process is meant to establish a firm foundation for new knowledge to be built upon. But numerous publishing and funding efforts - like arXiv, Fast Grants, or Sci-Hub, for example - have exposed the desire for science to go faster. This tension between fast and slow is as high as it's been since the emergence of popular science journals in the mid-nineteenth century. $\underline{1}$

Back then, the new system of journals emerged in parallel, outside the formality of the Academy, which still centered around the deliberations and books by members of the learned societies. Adaptation to the faster, more open system of journal publishing was a pragmatic evolution that took generations. We're living amidst a similar sort of dissonance. Formal progress towards open science has not kept pace with the emergence of informal tools developed outside the Academy that take full advantage of digital infrastructure and possibilities. These informal tools are the prototypes pointing us towards our knowledge futures.

One of those informal frontiers is crowdfunding for scientific research. For the past year, I've worked on Experiment, helping hundreds of scientists design and launch crowdfunding campaigns for their research questions. Experiment has been doing this for almost a decade, with more than $\underline{1}, 000$ successfully funded projects on the platform. The process is very different than the grant funding mechanisms set up by agencies and foundations. It's not big money yet, as the average fundraise is still $\sim \$ 5,000$. But in many ways, the process is better: faster, transparent, and more encouraging to early-career scientists. Of all the lessons learned, one stands out for broader consideration: grant proposals and processes should be open by default. Grant proposals that meet basic requirements for scientific merit and rigor should be posted online, ideally in a standardized format, in a centralized (or several) database or clearinghouse. They should include more detail than just the abstract and dollar amount totals that are currently shown now on federal databases, especially in terms of budgets and costs. The proposals should include a DOI number so that future work can point back to the original question, thinking, and scope. A link to these open grant proposals should be broadly accepted as sufficient for submission to requests from agencies or foundations.

This is standard operating procedure on Experiment. Every project that passes Experiment's merit and integrity benchmarks is given the chance to find a community 
of support for the work. The broader research ecosystem would be better off if this was adopted as standard operating procedure. Default open grant proposals would transform the funding ecosystem, with the benefits accruing to both individual scientists as well as the broader scientific community. Here's a list of improvements:

\section{Open proposals would make research funding project-centric, rather than}

funder-centric. The current gravitational center of academia is the funder, whether agency or foundation. Everyone in science recognizes these thoughts and statements: What project can I get funded? How can I shape my proposal to increase my funding prospects? Where can I publish for maximum visibility to improve my future funding opportunities? What are the funding deadlines and timelines?

With open proposals, because the funding could come from any number of different funders, there is a reduced tendency for the proposal to warp to match any individual funder's call. The result is less gamesmanship and more authentic curiosity. The transparency extends to project costs. One problem with a funder-centric ecosystem is that grant budgets often shrink or expand to fit the grant limits imposed by the funder, rather than reflecting the true cost of pursuing a research question.

Open proposals would promote more accurate budgets, and the transparency would provide a public cost accounting opportunity for the entirety of science to learn and build upon. At present, there is little downward pressure on the costs of scientific tooling and equipment precisely because budgets are opaque. Meanwhile, the cost of doing research directly impacts what gets studied and who does the studying. This is true in every discipline, from astronomy to marine microbiology. Cost is the driving factor in deciding what equipment a lab will buy or share. And it can significantly affect the pace of discovery. Opportunities to improve or disrupt expensive equipment bottlenecks would be made visible with proposal transparency.

Open proposals would increase the surface area of collaboration by allowing researchers to find potential partners earlier in the process or across social networks that they may not have been connected to before. Collaboration is a boon for ideas. Increasing the rate of sharing and cooperation among researchers is a proven route to improved output. Collaboration indirectly affects costs, too, as the most effective way to reduce the cost for scientific equipment is through sharing. That's how large telescopes are funded and financed, as well as costly underwater vehicles. There's an opportunity to extend this line of thinking to multiple scenarios and genres of scientific equipment, but it requires collaboration early on in the process. 
Open proposals would improve citation metrics. Brian Nosek and colleagues make a compelling case for the value of preregistration to improve the credibility of research findings. Open proposals are a more extreme form with all the accruing benefits. Making proposals an easily citable piece of content will improve their quality and rigor, as researchers expect a wider audience. The current publishing paradigm puts too much emphasis on results, which causes a raft of sub-optimal behaviors. Open proposals would create an opportunity to reward the best question-askers in addition to the best question-answerers.

Open proposals would give us a view into the whole of science, including the unfunded proposals and the experiments with null results. At present, this work usually doesn't see the light of day and we lose out on the opportunity to collectively learn from what hasn't worked.

So why not open proposals?

The first and major concern that scientists bring up: they'll be scooped! They worry an open proposal will allow someone else to catch wind of their line of thinking and research and beat them to publication. It's a natural reaction given the importance placed on priority.

However, the data doesn't justify the concern. Researchers have analyzed the effect of being scooped in the field of structural biology, an arena particularly well situated to study the effects because of the rewards of priority and the well-defined objectives for publication. They found that being scooped is not the end of the world. Scooped papers still receive, on average, $45 \%$ of the total future citations accrued by scooped and scooper. It's not winner-take-all. The scientists involved, however, were deeply concerned and fearful. A survey found their perception of the effects of being scooped to be much more severe than the data supported. Also, it should be reiterated that default open doesn't mean always open. If a scientist believes the risk of transparency is too detrimental to their prospects, especially if there's a need to protect patentable inventions, a traditional application process could still be arranged.

Despite all the upside and the early research on the effects of being scooped, it's hard to make a convincing argument for such a dramatic change. There is too much speculation, too little data, and plenty of good reasons to doubt. I have anecdotal hope, though. As I've explained this idea for consideration, I've gotten two dramatically different responses. Academic scientists are certain it won't happen and non- 
academics are surprised it hasn't already. In these scenarios, the delta is usually overcome through pragmatism.

This is the value and role of the informal tools and amateur scientists: to wander off the path and bring back anything useful. The utility of open proposals will be proven out (or not) on these informal frontiers. I welcome your participation and your skepticism. After all, it's always at the point of tension where our knowledge futures are forged.

\section{0 read more essays in the "Business of Knowing" summer series $\square$}

\section{Footnotes}

1. I recommend The Scientific Journal and Making "Nature" for more about the evolutionary history of the medium. $\doteq$ 